\title{
Pancreatic Islet-on-a-Chip: Examining the Associated Vascular Endothelial Cells in Microfluidic Culture.
}

\author{
J.V. Rocheleau,* \\ * Institute of Biomaterials and Biomedical Engineering, University of Toronto, Toronto Ontario, \\ Canada M5S 3G9; and Toronto General Research Institute, University Health Network, Toronto, \\ Ontario, Canada M5G 1L7
}

Endothelial cells (EC) are integral to the characteristically dense vasculature of pancreatic islets. This vasculature enables accurate blood-glucose sensing and rapid secretion of insulin into the blood stream. It also provides pro-survival hemodynamic signals to EC. How EC and beta cells interact to affect glucose stimulated insulin response is an actively debated topic. However, long-term studies in the ex vivo tissue are limited by the loss of EC over a period of days in traditional culture. We postulate that the EC die in part from an absence of the shear and media exchange provided by hemodynamic fluid flow. To test the role of hemodynamic forces on EC, we created a microfluidic device capable of supplying a range of fluid flow to ex vivo islets. Our protocol controls temperature, $\mathrm{pH}$ and bubble formation using two hot plates and a syringe pump for long-term desk top experimentation.

Using this microfluidic device with immunofluorescence microscopy, we examined the morphological response of islet-EC to a variety of flow rates for 24 and 48 hours. Our results show more than twice the average percent area and connectivity of EC in islets treated in the device as compared to no-flow controls stored in traditional cell culture. Using this device with varying media viscosity, we determined that the differences in morphology are due to media exchange and not shear-activated survival. Finally, using this device and live cell $\mathrm{Ca}^{2+}$-imaging, we observed a moderately dampened beta-cell glucose-stimulated response, but robust $\mathrm{KCl}$ response consistent with a reduced quality of the peripheral cells. We further used the microfluidic device to collect the glucose-stimulate insulin response and observed both normal glucose-stimulated response in the $48 \mathrm{hr}$ treated islets compared to no flow controls. These data are consistent with a normal glucosestimulated response from the central beta-cells of the flow-treated islets. Overall, our data indicates that flow in a microfluidic device provides a reliable co-culture environment enabling the long-term study of cell biology in the pancreatic islet. 